\title{
Relationship of resilience with occupational stress among nurses in coronavirus ward of Khatam Al-Anbia Hospital, Gonbad Kavous, 2020
}

\author{
Samira Khanmohammadi¹, Azam Hajibeglo², Maryam Rashidan¹ ${ }^{1}$ Karvan Bekmaz ${ }^{3}$ \\ ${ }^{1}$ Ali Abad Katoul Branch, Islamic Azad University, Ali Abad Katoul, Iran \\ ${ }^{2} S c h o o l$ of Nursing and Midwifery, Shahid Beheshti University of Medical Sciences, Tehran, Iran \\ ${ }^{3}$ Department of Nursing, Orumieh Branch, Islamic Azad University, Orumieh, Iran \\ Neuropsychiatria i Neuropsychologia 2020; 15, 1-2: 1-6
}

Address for correspondence:

Dr Karvan Bekmaz

Department of Nursing

Orumieh Branch

Islamic Azad University

Orumieh, Iran

e-mail: k.bekmaz@iaurmia.ac.ir

\begin{abstract}
Introduction: Occupational stress is one of the predictors of psychological and physical complications among nurses. High working pressure, lack of pharmacotherapy and the rate of transmission of coronavirus are the major causes of stress in nurses in coronavirus wards. Resilience is the process of coping with stressful events. The present study aims to investigate the relationship between resilience and occupational stress among nurses in coronavirus wards.

Material and methods: In this descriptive cross-sectional study, 150 nurses working in the coronavirus wards for at least one month were selected and examined using the available sampling method. The data collection instruments were the Demographic Questionnaire, the Connor and Davidson Resilience Questionnaire, and French's Expanded Nursing Stress Scale (ENSS).

Results: The mean and standard deviation of occupational stress level of nurses were $147.12 \pm 27.19$ and the mean and standard deviation of resilience rate of nurses were $67.43 \pm 10.25$. Spearman's correlation coefficient test showed a significant and inverse relationship between occupational stress and resilience of nurses $(r=-0.187, p=0.022)$.

Conclusions: The highest stressor for nurses in the coronavirus ward was the uncertainty about treatments, patients and their families. As nurses' resilience increases, so does their occupational stress. Resilience is a modifier of nursing occupational stress. To empower nurses against stress, it is recommended that resilience is taken into account in nursing education.
\end{abstract}

Key words: resilience, nurses, occupational stress, coronavirus ward.

\section{Introduction}

Nursing is the core of the health care system and plays an important role in community health. Nursing is one of the most stressful jobs and nurses are under severe occupational stress. Occupational stress is one of the most important factors in physical and psychological complications in personnel and reduction of the productivity of organizations (Bartlett et al. 2016). On the other hand, nurses working in special wards may be more stressed due to their specific working conditions and patients. Therefore, defined as the ability to overcome and master difficult situations in one's life, resilience can be helpful in this regard (Barlow and Zangaro
2010). Resilience is the ability or consequence of successful adaptation to threatening conditions. In other words, resilience is a positive adaptation to adverse conditions (Deldar et al. 2018). High resilience in nurses enables them to use positive adaptive skills in coping with stress and helps them keep up with the stressful environment of the special department. Also, high resilience is associated with increasing mental health and life satisfaction. Resilience is one of the characteristics that helps nurses adapt to the stressors of their working environment. Some researchers also believe that resilience capacity is essential for success in the nursing profession (Brown 2016). Warelow and Edward (2017) argue that 
in the $21^{\text {st }}$ century, nurses must skillfully develop resilience to deal with professional problems in order to overcome negative experiences and turn those experiences into positive ones.

At present, one of the main challenges of medical and hospital centers around the world is COVID-19 as a respiratory disease. The disease, caused by the new coronavirus, was first detected in Wuhan, Hubei Province, China, has affected several thousand people and is on the rise internationally - so much so that in March 2020, the World Health Organization identified COVID-19 as an epidemic worldwide (Yang et al. 2020).

The initial assumption is that the main transmission path of COVID-19 is airborne droplets that are thrown out by people coughing, sneezing or breathing. However, there are concerns that it could be transmitted by air. In the face of the COVID-19 crisis, staff in the healthcare sector, especially nurses and physicians, are at the forefront of coping and are exposed to a variety of psychological problems during diagnosis, treatment and care service provision (Chan et al. 2015).

Excessive working pressure, lack of personal protective equipment, extensive media coverage, lack of pharmacotherapy, and lack of adequate support are all factors that impose an increasing psychological burden on health care workers, especially nurses, so that they show symptoms such as depression, insomnia, anxiety, and severe stress (Kim 2018). Therefore, considering the importance of the issue, we decided to conduct a study aimed at investigating the relationship between resilience and occupational stress of nurses in the COVID-19 (coronavirus) ward in 2020 to improve the health programs for nurses and family using the results of the research.

\section{Material and methods}

This cross-sectional descriptive study was performed on 150 nurses in corona wards in Khatam Al-Anbia Hospital in Gonbad Kavous, Golestan Province from 5 January to 30 April 2020. The sample size of this study was calculated with the equation

$$
n=\frac{z_{\left(1-\frac{\alpha}{2}\right)}^{2} P(1-P)}{d^{2}}
$$

to be 147 at a significance level of $\alpha=0.05$, $Z_{975}=1.96, p=0.25$, and $d=0.077$. For further generalization, we considered 150 subjects and selected and examined them using the available sampling method. The inclusion criteria of this study included nurses who had worked in the corona ward for at least one month and were mentally healthy, and the exclusion criterion of the study was lack of interest in participation. Data collection tools include a demographic information form (age, gender, marital status, working position, level of education, employment status, work experience, income level, shifts and average monthly overtime hours). Connor and Davidson's resilience questionnaire, which has 25 items, uses a Likert scale with "completely true" (zero), "rarely true" (one), "sometimes true" (two), "often true" (three) and "always true" (four). The test score ranges between 0 and 100. Higher scores indicate greater resilience. Cronbach's $\alpha$ coefficient (0.93) was used to determine the scientific validity and reliability of the content. In the study by Connor et al. (2003), the content validity of the scale was examined, and was confirmed by Cronbach's $\alpha$ coefficient of 0.89 .

French's Expanded Nursing Stress Scale (ENSS) had 9 subscales of "fatality", "conflict with doctors", "insufficient emotional readiness", “conflict with colleagues”, "problems with nurses", "workload", "uncertainty about treatments", "patients and their families" and "discrimination". 57 items of the questionnaire were set on a five-point Likert scale, and the responses included: I have no stress at all (Bartlett et al. 2016), I have stress sometimes (Barlow and Zangaro 2010), I have stress often (Deldar et al. 2018), I have severe stress (Brown 2016), this position does not include my duties (Warelow and Edward 2007) and if you have not encountered such a situation. The test scores ranges between 0 and 285. Higher scores indicate more stress. Cronbach's $\alpha$ coefficient was used to determine the content validity and reliability. The results of the subscales were: patient suffering and death $=0.75$, workload $=0.75$, uncertainty about treatment $=0.74$, conflict with doctors $=0.75$, conflict with other nurses $=0.75$, insufficient readiness $=0.77$, lack of support $=0.76$, and the Cronbach's $\alpha$ for the whole instrument was 0.94 .

In Chakim and Erlen's (2007) study, the content validity of the scale was examined, and was confirmed by Cronbach's $\alpha$ coefficient of 0.85 , and Cronbach's $\alpha$ for the subscales was as follows: patient suffering and death $=0.746$, workload $=0.746$, uncertainty about treatment $=0.745$, conflict with doctors $=0.752$, conflict with other nurses $=0.749$, lack of adequate preparation $=0.767$, lack of support $=0.763$ (Cha et al. 2007). 
The face and content validity of these two questionnaires were confirmed by five members of the Educational and Clinical Nursing Research Committee of Khatam Al-Anbia Hospital in Gonbad Kavous. In this study, after the research plan was approved and the code of ethics was received from Golestan Social Security Organization, the researcher went to the Khatam Al-Anbia Hospital in Gonbad Kavous. The research unit and hospital officials were invited to participate in the study. Then, in the corona wards, the objectives of the research were explained to the nurses. Written consent was obtained from the nurses and they were assured that the study would be safe and that information would be kept confidential. The questionnaires were completed by the nurses of the corona wards. Completion of each questionnaire took 15-20 minutes, and at the time of completion of the forms by nurses, the researcher was present in the department answering the participants' questions about how to complete the questionnaire. The data were then entered into SPSS26 software and used at a significance level of 0.05 along with the Pearson correlation coefficient test, independent $t$-test and one-way analysis of variance.

\section{Results}

From among the 150 nurses, 64\% (96) were female, and $36 \%(54)$ were male, with an average age of $34.58 \pm 6.84$. In terms of marital status, it was found that $16 \%$ (24) were single and $84 \%$ (126) married. In terms of employment status, $65.3 \%$ (98) were official personnel, $23.3 \%$ (35) were contractual personnel, and $11.3 \%$ (17) were short term personnel. In terms of working position, $96 \%$ (144) were nurses, $4 \%(6)$ were head nurses and supervisors. $96 \%$ (144) had a bachelor's degree and 4\% (6) had a master's degree.

The average overtime for $47.3 \%$ (71) was between 50 and 100 hours, and the lowest, for $7.3 \%$ (11), was over 150 hours. In terms of work experience, the highest percentage of research units, $50.7 \%$ (76), was over 10 years and the lowest, 34\% (51), was less than 5 years.

The results of the study showed that the highest resilience score of the research units was 96 and the lowest score was 38 , with the mean and standard deviation being $67.43 \pm 10.25$, and the mean and standard deviation of stress in nurses were $147.12 \pm 27.19$, showing that the highest stress score of the research units was 216 and the lowest was 71 (Table 1).
The Spearman correlation coefficient test showed a significant and inverse relationship between nurses' resilience and their occupational stress $(r=-0.187, p=0.022)$, so that with increasing resilience, nurses' occupational stress decreases (Table 2).

Results of the study on nurses' resilience using demographic characteristics in the MannWhitney test showed no significant difference according to marital status ( $\phi=0.615)$, education $(p=0.249)$, or salary $(p=0.145)$. Also the independent $t$-test did not show a significant difference in the nurses' resilience considering gender $(p=0.415)$, position $(p=0.505)$ or shift $(p=0.440)$. The Kruskal-Wallis test did not show a significant difference in nurses' resilience in terms of employment status $(p=0.490)$ (Table 3).

Also, the Mann-Whitney test showed a significant difference according to nurses' stress and marital status $(\phi=0.045)$, and salary $(\phi=0.029)$. Also, the Kruskal-Wallis test showed a significant difference between nurses considering stress and employment status $(p=0.011)$. However, the Mann-Whitney test did not show a significant difference according to gender $(p=0.066)$, position $(p=0.799)$, education $(p=0.621)$, or shift $(p=0.04)$.

Table 1. Mean and standard deviation of dimensions of stress in nurses

\begin{tabular}{lc} 
Dimensions of stress in nurses & $\mathbf{M} \pm$ SD \\
Fatality & $18.11 \pm 3.65$ \\
\hline Conflict with doctors & $13.42 \pm 3.09$ \\
\hline Lack of emotional readiness & $7.26 \pm 1.95$ \\
\hline $\begin{array}{lc}\text { Problem in communicating with colle- } \\
\text { agues }\end{array}$ & $13.44 \pm 3.63$ \\
\hline $\begin{array}{l}\text { Problem in communicating with other } \\
\text { nurses }\end{array}$ & $18.77 \pm 4.22$ \\
\hline Workload & $22.73 \pm 4.95$ \\
\hline Uncertainty about treatment & $24.17 \pm 5.09$ \\
\hline Patients and their families & $24.08 \pm 4.91$ \\
\hline Discrimination & $9.15 \pm 2.58$ \\
\hline Total stress & $147.12 \pm 27.19$ \\
\hline
\end{tabular}

Table 2. Relationship between resilience, stress and overtime and age in nurses

\begin{tabular}{lccc} 
& Stress in nurses & Overtime & Age \\
Resilience & $r=-0.187$ & $r=-0.011$ & $r=-0.011$ \\
& $p=0.022$ & $p=0.890$ & $p=0.176$ \\
\hline
\end{tabular}


Table 3. Relationship between resilience and demographic characteristics in nurses

\begin{tabular}{|c|c|c|c|}
\hline \multicolumn{2}{|c|}{ Resilience/Demographic characteristics } & \multirow[t]{2}{*}{$M \pm S D$} & \multirow{4}{*}{$\begin{array}{c}p \text {-value } \\
0.415\end{array}$} \\
\hline Gender & & & \\
\hline Men & 54 & $68.33 \pm 11.42$ & \\
\hline Women & 96 & $66.90 \pm 9.73$ & \\
\hline \multicolumn{3}{|l|}{ Marital status } & 0.615 \\
\hline Single & 24 & $66.58 \pm 12.96$ & \\
\hline Married & 126 & $67.57 \pm 9.70$ & \\
\hline \multicolumn{3}{|l|}{ Position } & 0.505 \\
\hline Nurse & 144 & $67.30 \pm 10.21$ & \\
\hline Head nurse & 6 & $70.16 \pm 11.70$ & \\
\hline \multicolumn{3}{|l|}{ Education } & 0.249 \\
\hline Bachelor's degree & 144 & $67.63 \pm 10.00$ & \\
\hline Master's degree & 6 & $62.16 \pm 15.27$ & \\
\hline \multicolumn{3}{|l|}{ Employment status } & 0.490 \\
\hline Formal & 98 & $66.58 \pm 9.40$ & \\
\hline Short term & 35 & $69.91 \pm 9.48$ & \\
\hline Contractual & 17 & $67.11 \pm 15.28$ & \\
\hline \multicolumn{3}{|l|}{ Salary } & 0.145 \\
\hline Less than 5 million Tomans & 69 & $68.28 \pm 11.64$ & \\
\hline More than 5 million Tomans & 81 & $66.67 \pm 8.90$ & \\
\hline \multicolumn{3}{|l|}{ Shift } & 0.440 \\
\hline Fixed & 11 & $69.72 \pm 8.55$ & \\
\hline Variable & 139 & $67.23 \pm 10.37$ & \\
\hline
\end{tabular}

\section{Discussion}

The results showed that the average occupational stress is high in nurses. This study was performed on nurses in the corona wards, and considering that it is one of the wards that require taking special measures for patients, it can be considered as a special department, in line with the results of McCarthy et al. (2010). Occupational stress was significantly higher in the emergency department, intensive care unit, and pediatric wards (McCarthy et al. 2010). In line with the results of Sharma et al. (2014), there is a statistically significant relationship between workplace and stress levels. In this study, there were nurses who reported no resting time, among whom $42 \%$ suffered from moderate to severe stress, and nurses who felt that work was not boring had a lower stress than those who found work boring.

Nurses are at the heart of the treatment departments, and suffer high stresses. This study was performed on nurses and the results were in line with the results of the Sarafis et al. (2016) on medical team members, which showed that nurses were faced with a high level of stress. A study by Yuvanich et al. (2017) in five non-governmental hospitals in Thailand found that about $30 \%$ of nurses had moderate to severe stress. The results of Admi and Eilon-Moshe (2016) showed that Thai nurses have the highest levels of stress, the most important factor of which has been the lack of resources. Also, Lo et al. (2018) showed that the highest occupational stress rate could be found among nurses. Since the stress in nurses was a global concern in 2018, this study showed that recognizing this stress and adapting to it could prevent work withdrawal (Lo et al. 2018). A study by Bartlett et al. (2016) even showed that severe stress, anxiety, sleep disorders, and stress-related illnesses were more common in nursing students than students of other disciplines. In contrast to the results 
of this study, the results of the Divinakomar et al. (2014) study entitled "Occupational burnout, mental health, and occupational stress in public hospitals" found that the occupational stress in nurses is low. Also in the study of Chen and Fang (2016), who examined the job satisfaction of nurses and related factors in private hospitals, the occupational stress was investigated as a communication factor. They stated that the level of occupational stress among nurses was moderate (Azodo and Ezeja 2013). Due to the fact that patients in the corona wards have unpredictable conditions and nurses have high occupational conflicts due to their professional commitment, they suffer from high stress. The results of the study by Mark and Smith (2012) showed that high nurses' commitment and job engagement resulted in high stress, which was consistent with this study. The highest stress levels of nurses in the corona ward were related to the uncertainty about the treatments, and patients and their families, and the lowest stress levels were related to insufficient emotional readiness, as shown by Dagget et al. (2016), which was consistent with the study of the highest occupational stresses from uncertainty about treatment, i.e. $57.72 \%$. Also, the highest level of occupational stress due to mortality was $62.94 \%$ and workload $57.6 \%$, and the stress from sexual harassment stress had the lowest mean score of $46.19 \%$, which was inconsistent with the study (Dagget et al. 2016).

In the study of Bhatia et al. (2010), there was an inverse relationship between occupational stress and job satisfaction among nurses, and with increasing job satisfaction, their stress level decreased. Nurses with higher education and job positions, who have high levels of theoretical and clinical knowledge, show an ability to manage stress in clinical settings (Ren et al. 2018). A study on the relationship between occupational stress and demographic characteristics showed that there was no difference between men and women in terms of occupational stress. There was no difference in occupational stress between nurses with fixed shifts and those in comparison between the two groups. Incompatible with the results of this study, Romano $e t$ al. (2015) reported the highest levels of stress in female night shift nurses. A study by Lian and Tan (2014) showed that women generally experience more work stress than men. The correlation between stressors and coping strategies may depend on the interaction between employees and the type of problems they face (Lian and Tam 2014). A study by Lee et al. (2015) showed that men generally have more occupational stress than women. Regarding resilience, it was shown that the median resilience score is 67 , ranging from 0 to 100, which indicates the high resilience of nurses in the corona wards. In general, resilient individuals have the ability to recover and show higher optimism, flexible thinking skills, converting problems to learning opportunities, perseverance and endurance, sense of humor, and respectful behavior, problem solving and conflict resolution (Cooper et al. 2020). Inconsistent with the results of this study, Ren et al. (2018) showed that the mean score of overall resilience in nurses was significantly lower than in normal individuals. Reese et al. (2018) showed that nurses are less resilient in terms of occupational violence. Examining the relationship between nurses' resilience and demographic characteristics, the study showed that the level of education, marital status, gender, work experience and work shifts with resilience did not significantly differ. There was a significant relationship between nurses' resilience and age, years of experience, clinical rank and education (Leng et al. 2020). A study by Ren et al. (2018) showed that female nurses, with a high level of education and position and no night shifts, had high resilience. In the Lee et al. (2015) study, some demographic characteristics were related to nurses' resilience.

Occupational stress is also an important factor in explaining resilience. High levels of perceived occupational stress can reduce resilience. In this study, there was a significant and inverse relationship between occupational stress and resilience in nurses. In line with the results of this study, Ren et al. (2018) showed that resilience was also negatively associated with occupational stress. In a study by Zander $e$ al al. (2010), high occupational stress was related to low resilience in the pediatric oncology department. In a study by Miller et al. (2012), 22\% of nurses in the intensive care unit were highly resilient. The high resilience of these nurses was associated with the low rate of symptoms of anxiety, depression and occupational burnout.

\section{Limitations}

This study was performed only on nurses working in the corona wards of Khatam AlAnbia Hospital in Gonbad, and the viewpoints of nurses in other departments as well as nursing heads were not taken into account. This study was also performed in a short time. Therefore, it is recommended that studies be performed in other settings, taking into account the views of nursing heads, in a longer period of time. 


\section{Conclusions}

The most stressful dimension of the corona ward was the lack of confidence in the treatments, and patients and their families. No definitive cure has been found for COVID-19 yet, and this increases nurses' stress when working with corona patients and their families. Therefore, by increasing the resilience of nurses, their occupational stress can be reduced. Resilience is a modifier of stress among nurses. To empower nurses against stress, it is recommended that resilience be considered in nursing education.

\section{Acknowledgements}

I sincerely acknowledge all the research officials, authorities and nurses of Khatam Al-Anbia Hospital in Gonbad Kavous who cooperated in this study. This article is the result of approved research project No. 52897380 at Khatam Al-Anbia Hospital in Gonbad Kavous, and its funding has been provided by the same hospital.

\section{Disclosure}

The authors declare no conflict of interest.

\section{References}

1. Admi H, Eilon-Moshe Y. Do hospital shift charge nurses from different cultures experience similar stress? An international cross sectional study. Int J Nurs Stud 2016; 63: 48-57.

2. Azodo C, Ezeja E. Occupational stress among dental house officers and students in a tertiary healthcare centre. Odontostomatol Trop 2013; 36: 31-37.

3. Barlow KM, Zangaro GA. Meta-analysis of the reliability and validity of the Anticipated Turnover Scale across studies of registered nurses in the United States. J Nurs Manag 2010; 18: 862-873.

4. Bartlett ML, Taylor H, Nelson JD. Comparison of mental health characteristics and stress between baccalaureate nursing students and non-nursing students. J Nurs Educ 2016; 55: 87-90.

5. Bhatia N, Kishore J, Anand T, Jiloha RC. Occupational stress amongst nurses from two tertiary care hospitals in Delhi. Australasian Med J (Online) 2010; 3: 731.

6. Brown R. Determining the relationship among change fatigue, resilience, and job satisfaction of hospital staff nurses. South Dakota State University 2016.

7. Cha ES, Kim KH, Erlen JA. Translation of scales in cross-cultural research: issues and techniques. J Adv Nurs 2007; 58: 386-395.

8. Chan JF, Lau SK, To KK, et al. Middle East respiratory syndrome coronavirus: another zoonotic betacoronavirus causing SARS-like disease. Clin Microbiol Rev 2015; 28: 465-522.

9. Chen YM, Fang JB. Correlation between nursing work environment and nurse burnout, job satisfaction, and turnover intention in the western region of mainland China. Hu Li Za Zhi 2016; 63: 87-98.
10. Connor KM, Davidson JRT. Development of a new resilience scale: The Connor-Davidson resilience scale (CD-RISC). Depress Anxiety 2003; 18: 76-82.

11. Cooper AL, Brown JA, Rees CS, et al. Nurse resilience: A concept analysis. Int J Ment Health Nurs 2020; 29: 553-575.

12. Dagget T, Molla A, Belachew T. Job related stress among nurses working in Jimma Zone public hospitals, South West Ethiopia: a cross sectional study. BMC Nurs 2016; 15: 39 .

13. Deldar K, Froutan R, Dalvand S, et al. The relationship between resiliency and burnout in iranian nurses: a systematic review and meta-analysis. Open Access Maced J Med Sci 2018; 6: 2250-2256.

14. Kim Y. Nurses' experiences of care for patients with Middle East respiratory syndrome-coronavirus in South Ko rea. Am J Infect Control 2018; 46: 781-787.

15. Lee KJ, Forbes ML, Lukasiewicz GJ, et al. Promoting staff resilience in the pediatric intensive care unit. Am J Crit Care 2015; 24: 422-430.

16. Leng $M$, Xiu H, Yu P, et al. Current state and influencing factors of nurse resilience and perceived job-related stressors. J Contin Educ Nurs 2020; 51: 132-137.

17. Lian SY, Tam CL. Work stress, coping strategies and resilience: A study among working females. Asian Soc Sci 2014; 10: 41

18. Lo WY, Chien LY, Hwang FM, et al. From job stress to intention to leave among hospital nurses: A structural equation modelling approach. J Advanc Nurs 2018; 74: 677-688.

19. Mark G, Smith AP. Occupational stress, job characteris tics, coping, and the mental health of nurses. Br J Health Psychol 2012; 17: 505-521.

20. McCarthy VJ, Power S, Greiner BA. Perceived occupational stress in nurses working in Ireland. Occup Med 2010; 60: 604-610.

21. Rees C, Wirihana L, Eley R, et al. The effects of occupational violence on the well-being and resilience of nurses. J Nurs Adm 2018; 48: 452-458.

22. Ren Y, Zhou Y, Wang, S, et al. Exploratory study on resilience and its influencing factors among hospital nurses in Guangzhou, China. Int J Nurs Sci 2018; 5: 57-62.

23. Romano M, Festini F, Bronner L. Cross-sectional study on the determinants of work stress for nurses and intention of leaving the profession. Prof Inferm 2015; 68: 203-210.

24. Sarafis P, Rousaki E, Tsounis A, et al. The impact of occupational stress on nurses' caring behaviors and their health related quality of life. BMC Nurs 2016; 15: 56.

25. Sharma P, Davey A, Davey S, et al. Occupational stress among staff nurses: Controlling the risk to health. Indian J Occup Environ Med 2014; 18: 52-56.

26. Warelow P, Edward KL. Caring as a resilient practice in mental health nursing. Int J Ment Health Nurs 2007; 16: 132-135.

27. Yang Y, Peng F, Wang R, et al. The deadly coronaviruses: The 2003 SARS pandemic and the 2020 novel coronavirus epidemic in China. J Autoimmun 2020; 109: 102434.

28. Yuwanich N, Akhavan S, Nantsupawat W, et al. Experiences of occupational stress among emergency nurses at private hospitals in Bangkok, Thailand. Open J Nurs 2017; 7: 657-670.

29. Zander M, Hutton A, King L. Coping and resilience factors in pediatric oncology nurses. J Pediatr Oncol Nurs 2010; 27: 94-108. 